\title{
Agronegocio Conchero (Anadara tuberculosa): Aspectos socioeconómicos, Archipiélago de Jambelí
}

\section{Conchero Agribusiness (Anadara tuberculosa): Socio- economic aspects, Jambelí Archipelago}

Prado-Carpio, Eveligh Universidad Técnica de Machala (Machala - Ecuador) eprado@utmachala.edu.ec

Castro-Armijos, Cristhian Centro de especialización y capacitación agropecuaria CECAGROP (Ecuador)

Rentería-Minuche, Patricio Universidad Técnica de Machala (Machala - Ecuador)

Coronel-Reyes, Julian Centro de especialización y capacitación agropecuaria CECAGROP (Ecuador)

Paladines-Amaiquema, Jesser Universidad Técnica de Machala (Machala - Ecuador)

Martínez-Soto, Moisés Universidad del Universidad del Zulia (Zulia - Venezuela)

Revista Cumbres Vol.5 №2

Versión impresa ISSN 1390-9541

Versión electrónica ISSN 1390-3365

http://investigacion.utmachala.edu.ec/revistas/index.php/Cumbres 


\title{
RESUMEN
}

En este artículo, se presenta un estudio acerca de la parte socioeconómica del agronegocio en el sector conchero de la comuna Costa Rica, con el objetivo de diagnosticar la situación social y económica de los recolectores de concha prieta (Anadara tuberculosa). En este trabajo, se realizó una encuesta a 44 recolectores de la asociación de concheros Costa Rica, validando el instrumento con un Alpha de Cronbach de 0,87. Los resultados indican que existen gran participación del género femenino dedicado a la recolección del recurso conchero y que la mayoría de edad por participación de género esta entre 32-44 años, también hemos evidenciado que más de la mitad de las personas encuestadas no cuenta con algunos servicios básicos y de salud. El promedio de unidades de conchas prieta recolectadas por persona fue de 16.923 conchas al año, los costos e ingresos fueron de $\$ 836,00$ y $\$ 2.200$ anuales, respectivamente. Se aplicó una prueba Chi cuadrado obteniendo un p-valor 0,003 menor al alfa predefinido 0,05, según el cual se rechaza la hipótesis nula. Por esta razón, denotamos que existen indicios de una relación de dependencia entre las variables necesidad y oportunidad de emprender. La actividad del recurso conchero si genera utilidades para los comuneros.

Palabras clave: Concheros, cadena de valor, producción, innovación, equidad.

\begin{abstract}
In this article, we present a study about the socioeconomic part of the agribusiness in the shell sector of the commune Costa Rica, with the objective of diagnosing the social and economic situation of the collectors of concha prieta (Anadara tuberculosa). In this work, a survey was carried out to 44 collectors of the commune Costa Rica, validating the instrument with a Cronbach Alpha of 0.87 . The results indicate that there is great participation of the feminine gender dedicated to the collection of the conchero resource and that the majority of age for participation of gender is between 32-44 years, also we have evidenced that more than half of the people surveyed do not have some basic services and of health. The average number of black shell units collected per person was 16.923 per year, costs and income were $\$ 836,00$ and $\$ 2.200$ per year. A Chi-square test was applied obtaining a p-value of 0.003 (bilateral asymptotic sig.) that is compared with the Alpha of significance (assumed as 5\%), is too high to reject Ho, thus, it can be concluded that the variables are not related. The activity of the conchero resource does generate utilities for the comuneros.
\end{abstract}

Keywords: Fishing, value chain, production, innovation, equity. 


\section{INTRODUCCIÓN}

De los sectores alimentarios, la acuicultura es el más dinámico a nivel mundial, durante las últimas tres décadas ha tenido una tasa de crecimiento del 8,8\% (Rodríguez y Flores, 2014). En lo que respecta al periodo 2015 se obtuvo una producción estimada de 92,6 millones de toneladas métricas, cada país tiene establecida una zona económica exclusiva o de explotación de 200 millas náuticas a partir de las líneas base, pasado lo establecido la captura de las especies se considera libre ya que estas son aguas internacionales (Gálvez, 2016).

El sector pesquero es uno de los que presenta mayores problemas socioeconómicos en América Latina, sus niveles de sedentarismo y de bienestar son medios y bajos, esto puede verse reflejado en su salud mental y física (Véliz, Dörner, \& Ripoll, 2017).

Durante las últimas décadas, la captura de especies marinas ha venido en aumento, principalmente de bivalvos marinos (Steeves, Filgueira, Guyondet, Chassé, \& Comeau, 2018); sin embargo, desde la perspectiva de las ciencias sociales, la pesca continental ha sido muy poco estudiada, a pesar de tratarse de una actividad que aporta al bienestar y al desarrollo de las comunidades que habitan en las zonas costeras (Hernández, 2014).

La comunidad de pescadores pude llegar a ser un punto central en el proceso de desarrollo de la costa, sin embargo, para esto es fundamental fortalecer sus valores, a fin de que su gestión sea valorada, además de ello, una estrategia pudiera ser la dedicación a actividades múltiples que les permitan generar múltiples ingresos (Cahaya, 2015).

La Concha Prieta es un producto con gran potencial de desarrollo de mercados, pero que enfrenta serias imperfecciones y amenazas en su modelo de negocios, en los distintos eslabones de su cadena productiva, en virtud de las relaciones antagónicas y oportunistas entre los diferentes eslabones, el bajo nivel de innovación de sus procesos, su riesgosa calidad toxicológica y sanitaria, finalmente, la sobre explotación del medio natural, lo cual incluye a los concheros o pescadores (Prado-Carpio et al., 2018).

Dentro del archipiélago de Jambelí se localizan Puerto Bolívar, Puerto Jelí y Puerto Hualtaco, considerados como los puertos transcendentales de desembarque de la pesquería de la provincia de El Oro y del Ecuador. Estos puertos, entre los años 2008-2011 contribuyeron entre el 40 y 57\% del total de la pesquería (E. Mora et al. 2010).

La acuicultura pretende contribuir en el desarrollo de la población mediante los escenarios económico, ambiental y la capacidad de integrar a los diferentes sectores de la sociedad a la producción, sin afectar los ámbitos: sanitario, legal, logística de servicios, estructura del mercado local o regional.

Una investigación realizada en Costa Rica determinó la composición proximal de Anadara tuberculosa, resultando esta con niveles de proteína de $61,6 \%$, carbohidratos $21,6 \%$; cenizas, 8,8\%, lípidos 7,9 \%, el valor calórico fue de $5,2 \mathrm{kcal} / \mathrm{g}$., características que lo ubican como un producto recomen- 
dado para el consumo humano, por su bajo contenido de grasa y su alto contenido proteico (Prado-Carpio et al., 2018).

Según IICA (2010) Un agronegocio es un sistema integrado de negocios enfocado en el consumidor, que incluye los aspectos de producción primaria, procesamiento, transformación y todas las actividades de almacenamiento, distribución y comercialización, así como los servicios, públicos y privados, necesarios para que las empresas del sector operen competitivamente. Contraria a la visión tradicional, esta visión de los agronegocios considera a la agricultura como un sistema de cadenas de valor que se centra en dar satisfacción a las demandas y preferencias del consumidor, mediante la incorporación de prácticas y procedimientos que incluyen todas las actividades dentro y fuera de la unidad de producción.

Según Prado-Carpio et al., (2018), la mejora en la organización y de los procesos administrativos de los concheros de la comuna Costa Rica desarrollará el emprendimiento llevándolos a dueños de sus propias negociaciones y microempresarios de sus propias actividades económicas, mejorando su capacidad de negociación. Es importante la innovación ya que desarrollaran estrategias de mejoras en sus agronegocios, aplicando técnicas de inocuidad, un sistema de buenas prácticas y nuevos procesamientos agregando valor al producto haciendo más vistoso a nivel nacional e internacional. Así mismo es importante la parte ambiental utilizando tácticas que tiendan a reservar la superficie del ecosistema manglar y evitar la extracción de la concha prieta inferior a 4,5 cm.

Es por esto que se plantea como objetivo caracterizar social y económicamente el agronegocio de los capturadores de concha en el archipiélago de Jambelí, provincia de El Oro.

La investigación se realizó en el Archipiélago de Jambelí, en la provincia de El Oro, en la comuna Costa Rica; el diseño aplicado fue un diseño metodológico descriptivo y transversal, para lo cual, se diseñó un cuestionario conformado por tres ítems conformados por preguntas abiertas y cerradas. El tamaño de la muestra a encuestar fue de 44 extractores de concha que forman parte de la asociación de concheros Costa Rica.

El trabajo realizado está estructurado de la siguiente manera: introducción, materiales y métodos, resultados, conclusiones y referencias bibliográficas.

\section{MATERIALES Y MÉTODOS}

El presente estudio se desarrolló en el Archipiélago de Jambelí ubicado al suroeste de la provincia de El Oro, litoral sur del Ecuador centrando nuestra investigación se centra en la comuna Costa Rica, en donde mantiene en custodia 889 ha. de manglar, cuentan con 102 habitantes 153,29ha. de superficie.

La presente investigación tiene como finalidad un diseño metodológico descriptivo y transversal, es decir, busca determinar, evaluar e interpretar las variables categóricas y cuantitativas. Por lo cual, se diseñó un cuestionario conformado por tres ítems (tabla 1) que tiene preguntas abiertas y cerradas. 


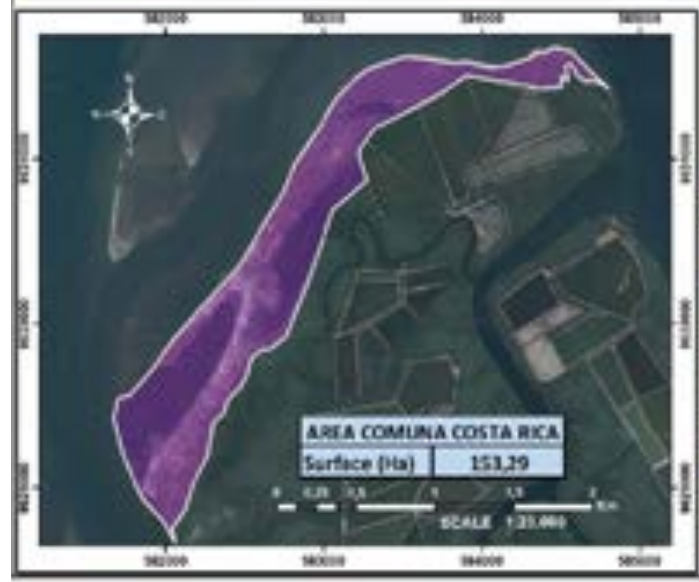

Figura No. 1: Comuna Costa Rica

Tabla 1. Aplicabilidad de un cuestionario a $n=44$ personas de la comuna Costa Rica, archipiélago de Jambelí, provincia El Oro.

\begin{tabular}{|l|l|}
\hline Ítem & Variables \\
\hline Identificación & Información demográfica y ubicación de la isla \\
\hline Económico & $\begin{array}{l}\text { Cantidad de concha prieta recolectada/año, costos producción/año, ingresos/ } \\
\text { año }\end{array}$ \\
\hline Social & $\begin{array}{l}\text { Nivel de estudio, servicios básicos, seguridad social, equidad de género, razón de } \\
\text { emprender en un agronegocio. }\end{array}$ \\
\hline
\end{tabular}

El cual fue validado por un panel de 5 experto del sector conchero, utilizando la técnica del Alpha de Cronbach del cual se obtuvo un 0,87. En investigaciones relacionadas con datos cualitativos similares al estudio, Sánchez (2018) obtienen un Alpha de 0,814 y (Alonso, Díaz, \& Mas, 2017) 0,75 recomendando el uso de valores a partir 0,70 con lo que se aceptó el modelo de encuesta. Definido las variables y el instrumento de evaluación, se tomó toda la población de 44 personas, el registro se obtuvo de la junta parroquial de Jambelí.

La información obtenida fue tabulada en una base de datos en Excel, posteriormente se procesó a través del programa estadístico SPSS Versión 24 para su análisis descriptivo y aplicar una prueba Chi cuadrado. Con base a los resultados obtenidos se elaboraron gráficas y tablas para su respectiva interpretación.

\section{Población y muestra}

La población a encuestar fue de 44 recolectores de concha de la isla Costa Rica, provincia de El Oro.

\section{RESULTADOS}

De acuerdo a los resultados, en la figura 2, se ha evidenciado más de la mitad de habitantes de la comuna Costa Rica son hombres (91.11\%), en cambio el sexo femenino representa el (8.89\%) lo cual suma un 100\%. Estos datos se los obtuvo mediante una encuesta. 
En el análisis de los resultados en la figura 1b establece, que edades en el rango de 32-44 años, representa (48.89\%) del total de la distribución de edades de los recolectores del sitio Costa Rica, de los cuales un 40,09\% son hombres y un (8.8\%) son mujeres de dicho subtotal, esto último son los aportes relativos de los géneros a la tasa de participación relativa de dicho rango de edad (32-44 años).

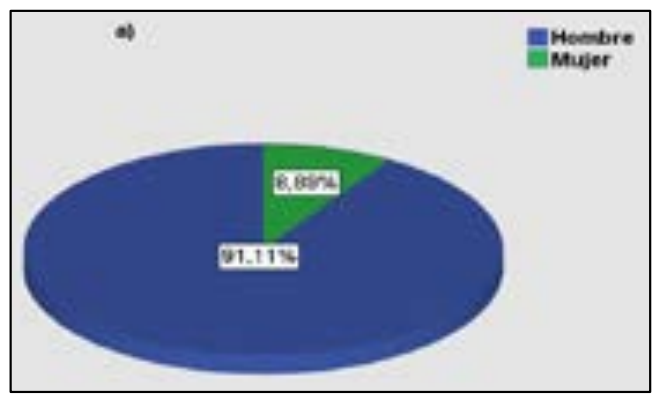

Figura 2.

a) Participación de género.

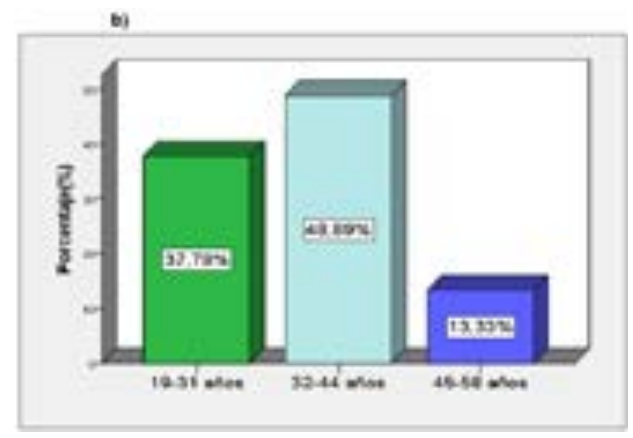

b) Participación por edad de los recolectores del sitio Costa Rica

Tabla 2. Resumen porcentual de variables sociales de los comuneros Costa Rica(n=44).

\begin{tabular}{|l|l|l|}
\hline Variable Sociales & Afirmación (\%) \\
\hline Energía Eléctrica & $\mathrm{Si}=100$ & $\mathrm{No}=0$ \\
\hline Alcantarillado & $\mathrm{Si}=100$ & $\mathrm{No}=0$ \\
\hline Agua potable & $\mathrm{Si}=100$ & $\mathrm{No}=0$ \\
\hline Seguro Social & $\mathrm{Si}=0$ & $\mathrm{No}=100$ \\
\hline Tv y radio & $\mathrm{Si}=75$ & $\mathrm{No}=25$ \\
\hline Telefonía fija & $\mathrm{Si}=0$ & $\mathrm{No}=100$ \\
\hline Telefonía móvil & $\mathrm{Si}=100$ & $\mathrm{No}=0$ \\
\hline Internet & $\mathrm{Si}=88$ & $\mathrm{No}=12$ \\
\hline
\end{tabular}

$\mathrm{Al}$ analizar las variables sociales en la figura 2, se aprecia que los comuneros no cuentan con seguro social, además, también carecen de telefonía fija. También se logró evidenciar que todas las personas contestaron que disponen de alcantarillado, energía eléctrica y agua potable. Más de la mitad utilizan los servicios de telefonía móvil, internet y radio.

Tabla 3. Nivel de instrucción por género.

\begin{tabular}{|l|l|l|l|l|}
\hline Sexo & Primaria & Ciclo Básico & Diversificado & Total \\
\hline Masculino & $45,94 \%$ & $15,35 \%$ & $0,00 \%$ & $56,70 \%$ \\
\hline Femenino & $21,42 \%$ & $17,29 \%$ & $0,00 \%$ & $43,30 \%$ \\
\hline
\end{tabular}

Se visualiza el nivel de estudio por género, en la tabla 3, de acuerdo a la encuesta aplicada el sexo masculino presenta un mayor porcentaje $(45,94 \%)$ que el sexo femenino (21.42\%). Otro escenario analizar es el nivel de estudio 
ciclo básico el sexo femenino está por encima con 15,00\% mientras que el sexo masculino representa el $17,29 \%$ y finalmente ninguna persona ha culminado el ciclo diversificado.

Tabla 4. Resumen descriptivo de producción, costos e ingresos

\begin{tabular}{|l|l|l|l|l|}
\hline \multirow{2}{*}{ Variables 1 } & Descriptivos 2 & \multicolumn{3}{|l|}{} \\
\cline { 2 - 5 } & Frecuencia & Media & Máximo & Mínimo \\
\hline *CCRA & & 16.923 & 75.200 & 10.950 \\
\hline *Costos (\$) & 44 & 836,00 & 901,55 & 540,93 \\
\hline *Ingreso (\$) & 44 & 2.200 & 2.372 & 1.423 \\
\hline
\end{tabular}

${ }^{1}$ Cantidad de Conchas recolectadas anual/100; costos de producción/año; Ingresos/año

${ }^{2} \mathrm{~N}$ (numero), (Media), Min (Mínimo); Max (Máximo).

Se evidencia los resultados de la producción, los costos de producción e ingresos anuales en la tabla 4, donde la media de conchas prieta recolectadas por persona fue de 16.923 conchas al año, de acuerdo a datos del GEOSGES, (2016) el promedio fue de 15.680 conchas, es decir el rendimiento ha tenido un incremento, esto se debe a la plan de reforestación implementado por el gobierno ecuatoriano (Beitl, 2014).

En cuanto a los costos de producción el valor promedio anual fue de \$ 836,00 mientras que los ingresos monetarios ascienden a \$ 2.200 dólares, es decir, si generan utilidades dedicándose a la actividad de recolección de concha prieta.

Tabla 5. Tabla de contingencia de género y razón de emprender en un agronegocio.

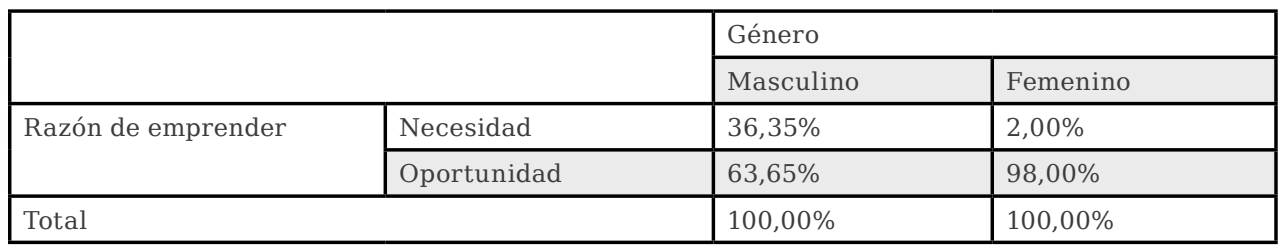

Los resultados del presente trabajo en tabla 5, revelan que, del total de encuestados, se logró constatar que la necesidad de emprender es del $2 \%$, sin embargo, la mayoría (98\%) del género femenino establecen como oportunidad de emprender en un agronegocio, coincidiendo con lo planteado por (Prado-Carpio et al., 2018). Así mismo, el género masculino-oportunidad de emprender es de $(63,65 \%)$, otro escenario es la necesidad de emprender que es de $(36,35 \%)$. Esto indica un panorama favorable para la implementación de un agronegocio.

Se ha denotado claramente en la tabla 6 que se obtuvo p-valor (Sig. asintótica bilateral) 0,003 menor al alfa predefinido 0,05, según el cual se rechaza la hipótesis nula. Por esta razón, denotamos que existen indicios de una relación de dependencia entre las variables necesidad y oportunidad de 
emprender, por lo tanto, se puede concluir con un nivel de confianza de $95 \%$ que ambas variables son dependientes.

Los resultados alcanzados indican claramente, que al aplicar una encuesta a 44 personas, se establece que el $100 \%$ de ellos no cuenta con un seguro social (tabla 2), por lo cual, es necesario contar con un servicio de salud disponible, según Aguilar, Bozada, \& López, (2017); Díaz \& Mera, 2017) mejora la calidad vida porque se conoce el historial clínico del paciente y se podría prevenir futuras enfermedades.

Es importante señalar, que las personas encuestadas mencionaron que no han finalizado sus estudios secundarios (tabla 3), según Domínguez et al., (2017) estos resultados no permitirán el desarrollo de la comunidad debido a su bajo de nivel de instrucción.

De acuerdo a los resultados alcanzados son muy similares a los obtenidos por Prado-Carpio et al., (2018); León Serrano (2017) en donde se pudo concluir una alta dependencia entre la equidad de género y los aspectos como necesidad y oportunidad para emprender, es decir existe dependencia entre las variables.

Tabla 6. Prueba Chi-Cuadrado

\begin{tabular}{|l|l|l|l|}
\hline Variables & Valor & gl & Significación asintótica (bilateral) \\
\hline Chi-cuadrado de Pearson & $7,150 \mathrm{a}$ & 1 & 0,003 \\
\hline N de casos válidos & 44 & & \\
\hline
\end{tabular}

\section{CONCLUSIONES}

- Más de la mitad de las personas encuestadas no cuenta con algunos servicios básicos y de salud, entre ellos, alcantarillado, telefonía fija y seguro social.

- Existe participación del género femenino dedicado a la recolección del recurso conchero.

- El nivel de instrucción es muy bajo entre los comuneros.

- Los resultados alcanzados mediante la prueba Chi cuadrado, establecen que existen indicios de una relación de dependencia entre las variables necesidad y oportunidad de emprender, de esta manera, se puede concluir que las variables están relacionadas.

\section{REFERENCIAS BIBLIOGRÁFICAS}

Aguilar, M. E. F., Bozada, G. T., \& López, B. M. P. (2017). Causas frecuentes de cirrosis hepática en el hospital ambulatorio, seguro social, jipijapa, manabí, ecuador. . ISSN 2602-8166. Retrieved from http://ojs.unesum.edu.ec/unesum/index.php/unesumciencias/article/ view/79 
Alonso, Á. V., Díaz, J. A. A., \& Mas, M. A. M. (2017). Aplicación del cuestionario de opiniones CTS con una nueva metodología en la evaluación de un curso de formación CTS del profesorado. Tarbiya, Revista de Investigación E. Retrieved from https://revistas.uam.es/tarbiya/article/view/7216

Beitl, C. M. (2014). Navigating Over Space and Time: Fishing Effort Allocation and the Development of Customary Norms in an Open-Access Mangrove Estuary in Ecuador. Human Ecology, 42(3), 395-411. https://doi.org/10.1007/s10745-014-9655-7

Cahaya, A. (2015). Fishermen Community in the Coastal Area: A Note from Indonesian Poor Family. Procedia Economics and Finance, 26(15), 29-33. https://doi.org/10.1016/S2212-5671(15)00801-1

Díaz, N. S. V., \& Mera, J. T. (2017). Institucionalidad del iess instituto ecuatoriano de seguridad social. Espirales revista multidisciplinaria de investigación, 1(1). https://doi.org/10.31876/re.v1i1.8

Domínguez, Y. M., Gutiérrez-Barroso, J., \& Gómez-Galdona, N. (2017). Equidad, Género Y Diversidad En Educación. European Scientific Journal, ESJ, 13(7). https://doi.org/10.19044/esj.2017.v13n7p\%p

Hernández, A. (2014). Recovery and importance of fishing and fishermen in Lake Chapala. Cultural, IV(1), 237-240. Retrieved from http://www.scielo. org.mx/scielo.php? script=sci_arttext\&pid=S1870-11912016000100237

León Serrano, L. A. (2017). La equidad de género en los emprendimientos. Fides et Ratio-Revista de Difusión Cultural Y Científica de La Universidad La Salle En Bolivia, 13(13), 107-125. Retrieved from http://www.scielo.org.bo/scielo. php?pid=S2071-081X2017000100008\&script=sci_abstract\&tlng $=$ en

Prado-Carpio, E., Castro-Armijos, C. J., Castillo-Herrera, S., Quezada-Abad, C., Coronel-Reyes, J., \& Arias-Chiriboga, H. (2018). La Equidad De Género En Los Agronegocios: Sector Comercio De Insumos Agropecuarios Y Servicios Veterinarios Del Cantón Machala. European Scientific Journal, ESJ, 14(8). https://doi.org/10.19044/esj.2018.v14n8p\%p

Prado-Carpio, Eveligh, César Quezada-Abad, Moisés Martínez-Soto, Carlos Rodríguez-Monroy, y Anne Morris-Díaz. (2018). "An Approximation to Agribusiness Development in the Value Chain of the Bivalve Mollusk" Anadara Tuberculosa (Sowerby, 1833)(Arcidae)"». Proceedings in Food System Dynamics, 382-93. http://centmapress.ilb.uni-bonn.de/ojs/index. php/proceedings/article/view/1831.

Prado-Carpio, E., Martinez-Soto, M., Rodriguez-Monroy, C., \& Morris, A., Rodriguez-Monrroy C., Borja-Herera, A., (2018). Modelo Teórico de Relaciones entre la Gestión de Agronegocios y el Desempeño de la Cadena de Valor de la Concha Prieta “ Anadara tuberculosa ," 16(January), 1-9. https://doi.org/10.18687/LACCEI2018.1.1.396

Sánchez, E. R., \& Pedreño, M. H. (2018). Análisis de las causas endógenas y exógenas del abandono escolar temprano: una investigación cualitativa. Educación XX1, 0(0). https://doi.org/10.5944/educxx1.21351

Serrano, L. A. L. (2017). La equidad de género en las decisiones de consumo y ahorro de las familias parroquia el retiro, provincia de el oro. revista ciencias pedagógicas e innovación, 5(1). https://doi.org/10.26423/rcpi.v5i1.158 
Steeves, L. E., Filgueira, R., Guyondet, T., Chassé, J., \& Comeau, L. (2018). Past, Present, and Future: Performance of Two Bivalve Species Under Changing Environmental Conditions. Frontiers in Marine Science, 5(May 2018), 1-14. https://doi.org/10.3389/fmars.2018.00184

Mora, Elba, Juan Moreno, and Viviana Jurado. 2009. “La Pesquería Artesanal Del Recurso Concha En Las Zonas de Esmeraldas Y El Oro, Durante El 2008." http://www.oceandocs.org/bitstream/handle/1834/4563/ doc\%202.\%20la\%20pesquer\%cda\%20artesanal\%20del\%20recurso\%20 concha $\% 20$ en $\% 201$ as $\% 20$ zonas $\% 20$ de $\% 20$ esmeraldas $\% 20 y \% 20$ el\%20 oro,\%20durante\%20el\%202008.pdf?sequence=1.

Mora, E., J. Moreno, V. Jurado, and L. Flores. (2010). “La Pesquería de La Concha Prieta (Anadara Tuberculosa Y Anadara Similis) En El 2009: Indicadores Pesqueros Y Condición Reproductiva En La Zona Sur Y Norte de Ecuador." INP (Ecuador). https://www.oceandocs.org/bitstream/handle/1834/4795/3.\%20la\%20pesqueria\%20de\%20la\%20concha\%20prieta\%20\%20e.\%20mora_2010\%20_1_.pdf?sequence=1.

Rodríguez, H. \& A. Flores. (2014). Acuicultura de pequeña escala y recursos limitados en América Latina y el Caribe. Hacia un enfoque integral de políticas públicas. FAO, Rome, $94 \mathrm{pp}$.

Véliz, A., Dörner, A., \& Ripoll, M. (2017). Bienestar sicológico y nivel de sedentarismo de pescadores artesanales de la Región de Los Lagos, Chile. Humanidades Médicas, 17(2), 323-337. Retrieved from http://scielo.sld.cu/ scielo.php?script=sci_arttext\&pid=S1727-81202017000200006 